\title{
Postural function in idiopathic normal pressure hydrocephalus before and after shunt surgery: a controlled study using computerized dynamic posturography (EquiTest)
}

\author{
Fredrik Lundin, Torbjörn Ledin, C Wikkelsø and Göran Leijon
}

\section{Linköping University Post Print}

\section{Tweet}

N.B.: When citing this work, cite the original article.

Original Publication:

Fredrik Lundin, Torbjörn Ledin, C Wikkelso and Göran Leijon, Postural function in idiopathic normal pressure hydrocephalus before and after shunt surgery: a controlled study using computerized dynamic posturography (EquiTest), 2013, Clinical neurology and neurosurgery (Dutch-Flemish ed. Print), (115), 9, 1626-1631.

http://dx.doi.org/10.1016/j.clineuro.2013.02.015

Copyright: Elsevier

http://www.elsevier.com/

Postprint available at: Linköping University Electronic Press

http://urn.kb.se/resolve?urn=urn:nbn:se:liu:diva-100038 


\section{Postural Function in \\ Idiopathic Normal Pressure Hydrocephalus \\ Before and After Shunt Surgery \\ A Controlled Study Using \\ Computerized Dynamic Posturography (EquiTest)}

Lundin $F^{1}$, Ledin $T^{2}$, Wikkels $\phi C^{3}$, Leijon $G^{1}$

1. Department of Clinical and Experimental Medicine, Faculty of Health Sciences, Linköping University, Sweden and Department of Neurology, County Council of Östergötland, Linköping, Sweden.

2. Department of Clinical and Experimental Medicine, Faculty of Health Sciences, Linköping University, Sweden and Department of Otorhinolaryngology, County Council of Östergötland, Linköping, Sweden.

3. Institute of Neuroscience and Physiology, Sahlgrenska Academy, University of Gothenburg, Sweden Corresponding Author: Fredrik Lundin

Address: Department of Neurology, University Hospital, S-581 85 Linköping,

Mail: Fredrik.Lundin@lio.se

Telephone: +46101034415

Fax: +46101034438

Key words: idiopathic Normal Pressure Hydrocephalus, Postural Function, Computerized Dynamic Posturography, Shunt surgery

Abstract words: 277

Article words: 2750 


\begin{abstract}
Introduction

Postural dysfunction is one of the major features of idiopathic Normal Hydrocephalus (iNPH). With computerized dynamic posturography (CDP) balance can be assessed objectively. The primary aim of this study was to describe the postural function in iNPH patients pre- and post-operatively in comparison with healthy individuals (HI) using CDP.
\end{abstract}

\title{
Subjects and methods
}

Thirty-five patients (16 M, 19 F) with a mean age of 73 (range 49-81) with iNPH, and sixteen HI (7 M, 9 F) aged 73 (62-89) were included. iNPH patients were operated on with a ventriculo-peritoneal shunt. Patients and HI were tested regarding motor function, balance and cognition. CDP, EquiTest (NeuroCom International, Clackamas, OR), was performed before and three months after shunt surgery and twice in HI, with a three-month interval.

\section{Results}

Pre-operatively, the 35 patients had poorer balance measured with the Sensory Organizing Test (SOT) score in every condition ( $\mathrm{p}=0.01$ in SOT 1 and $\mathrm{p}<0.001$ in SOT $2-6$ ) compared to the HI. The greatest difference was in test conditions measuring mainly vestibular function, where loss of balance (LOB) was frequent. Twenty patients were evaluated three months after shunt surgery and 18/20 (90\%) of them were considered shunt responders, with a mean improvement of motor score of 26\% (range 5-67 $\%)$. There was an improvement post-operatively in the weighted composite SOT score $(\mathrm{p}<0.05)$ but no significant change in any of the SOT conditions. LOB was not significantly reduced in any of the test conditions.

\section{Conclusion}


CDP showed that the patients had a poorer balance than the HI. The greatest difference was in SOT 56 , indicating that the postural disturbance is of primarily central vestibular origin. There was a slight improvement of balance post-operatively. 


\section{Introduction}

Postural disturbance is an important symptom of idiopathic Normal Pressure Hydrocephalus (iNPH) and is, together with the motor dysfunction, responsible for the most striking symptom, namely gait difficulties (1). The other classical symptoms of iNPH are impaired cognition and urinary urgency (1). The symptoms usually exist together, but the severity may range from subtle to disabling (2). iNPH usually affects older individuals with a mean age of around 70 years $(3,4)$ and the prevalence has been estimated to be as high as $1.4 \%$ in elderly Japanese people (5). iNPH is characterized by a ventricular enlargement secondary to a cerebrospinal fluid (CSF) disturbance, where the CSF-pressure is within the normal limits. There have been a few studies addressing the issue of postural dysfunction in iNPH. Soelberg-Sörensen et al found a decreased postural stability (6) and Blomsterwall et al. went further and concluded that postural dysfunction is partly responsible for the gait difficulties, and that improvement after shunt surgery is more profound in tests assessing balance (7). In a later study, Blomsterwall et al. used a force platform to assess balance in patients with normal pressure hydrocephalus (NPH) and subcortical arteriosclerotic encephalopathy. In this study, no difference regarding balance could be detected between the groups of patients, but compared to HI the hydrocephalic patients had significantly larger sway area and showed higher backward velocity (8).

Postural function is dependent on vision, peripheral vestibular sense, proprioception and a central integration. With advancing age there is impairment in these functions, and if there is a selective impairment in one of them, greater demands are placed on the remaining functions in order to maintain a good balance (9). It is of great importance to assess balance in the elderly properly to identify individuals at risk of falling, since the consequences of traumatic injuries can be enormous, both for the individual and also for society.

There are many bedside clinical tests for assessing balance. The oldest, and one that is still performed, is Romberg's test (10), Other examples are the Tinetti Balance and Gait test (11) and the Berg Functional Balance Scale(12). They are easy to perform but have the problems of ceiling effects and poor specificity (13). 
Instrumental methods have been developed for assessing balance more accurately. Computerized dynamic posturography (CDP) is a method involving the measurement of ground reaction forces from which the centre of pressure and sway may be calculated (14). The simplest equipment is a force plate. A more advanced equipment is EquiTest (version 4.04 NeuroCom International, Clackamas, OR); a diagnostic tool that measures the sway in several conditions. CDP can potentially differentiate between different causes of postural dysfunction, such as vestibular, proprioceptive and visual. The method has never been used before to evaluate patients with hydrocephalus.

The primary aim of this study was to describe the postural function in iNPH patients by CDP pre-and post-operatively and in comparison with $\mathrm{HI}$.

\section{Subjects and Methods}

Thirty-five patients (16 males, 19 females) with mean age 73 (49-81) ( Table I) diagnosed as probable iNPH with modified iNPH guidelines (1) were consecutively included. They were recruited from the outpatient clinic of Neurology, University of Linköping.

Clinically there had to be a gait disturbance affecting both legs, including difficulties with tandem walking, multistep turning, decreased step length and a straddled gait where no other condition could be the cause. Patients with neurological symptoms of cortical origin, such as aphasia, apraxia and agnosia, were excluded. Decreased cognitive function and urgency of micturition might exist.

Radiologically, a symmetrical communicating ventricular dilatation without cortical infarcts or other lesions of clinical importance except lacunar infarcts $(<1 \mathrm{~mL})$, Evans index $\geq 0.3$, relative enlargement of temporal horns and third ventricle had to be present. Moderate cortical atrophy and moderate sub cortical ischemic white matter hyperintensities were accepted.

Patients with ICP > $18 \mathrm{mmHg}$, cerebrospinal fluid (CSF) changes compatible with a secondary normal pressure hydrocephalus, difficulties in handling the tests, or short expected survival time were excluded 


\section{Healthy Individuals}

Sixteen HI (7 males, 9 females) aged 73 (62-89) were consecutively recruited mainly from relatives and friends to staff members (Table I). They were subjectively healthy and had a normal gait, balance and cognition on examination. Medication and diseases not impairing gait and cognitive status were not considered reasons for exclusion.

\section{Clinical assessment}

The patients were assessed neurologically (FL), and an MRI of the brain was performed. A physiotherapist assessed the motor function by using the following tests: time needed for a 10-metre walk in seconds (w10mt) and number of steps (w10ms) at a self-selected speed and with their usual walking aid (8). A timed up and go test measuring seconds (TUGt) and steps (TUGs). This is a timed test for standing up from a chair, walking three metres, turning and walking back to the chair and sitting down(15). For balance, Romberg's test, modified after Blomsterwall et al., was used. It was performed standing, with the feet together, eyes closed and hands on the chest. Seconds to correction up to 60 seconds were registered (8). An occupational therapist performed cognitive testing with MMSE (16). CSF pressure was measured at a lumbar puncture. Cells and proteins were analysed in order to exclude patients with secondary NPH

The patients received a programmable Codman-Hakim (Codman/Johnson \& Johnson, Raynham, MA) ventriculo-peritoneal shunt ( $\mathrm{n}=31)$ with an opening pressure of mean $110(60-150) \mathrm{mm} \mathrm{H}_{2} \mathrm{O}$ or a fixed pressure valve, medium high, Codman- Hakim (Codman/Johnson \& Johnson, Raynham, MA) $(n=3)$. One patient did not undergo shunt surgery because the pre-operative examination indicated an unfavourable prognosis according to a consensus discussion. The patients were re-examined three months after surgery. Improvement was defined as a 5\% improvement in a motor score (MOS). MOS was calculated as a composite score with the percentage change of each item expressed as follows: $(\Delta \mathrm{w} 10 \mathrm{mt}+\Delta \mathrm{w} 10 \mathrm{~ms}+\Delta \mathrm{TUGt}+\Delta \mathrm{TUGs}) / 4$. 
Shunt dysfunction was considered if the patient did not reach the pre-defined improvement in MOS. A computer tomography of the brain and a plain radiograph were carried out, and if there were still doubts about the feasibility of a working shunt a CSF-dynamic test was performed. There were no adjustments made during the first three months after surgery.

The HI were examined for time needed for a 10-metre walk in seconds and number of steps at a selfselected speed. Romberg's test for assessing balance and an MMSE for evaluating cognitive status were performed.

\section{Computerized Dynamic Posturography}

Computerized dynamic posturography (CDP), EquiTest (version 4.04. NeuroCom International, Clackamas, OR) was used in which the patient stands on a dual force plate and a visual surround is enclosed. The feet were placed straight ahead with a distance of $15 \mathrm{~cm}$ between. The platform measures the force between the ground and the feet in a horizontal antero-posterior direction and from that the sway can be estimated. (NeuroCom International, Clackamas, OR) Patients and $\mathrm{HI}$ are examined in six separate conditions (Sensory organizing test, SOT); 1.Eyes open, fixed surface and visual surround. 2. Eyes closed, fixed surface. 3. Eyes open, fixed surface, sway referenced visual surround. 4. Eyes open, sway referenced surface, fixed visual surround. 5. Eyes closed, sway referenced surface. 6. Eyes open; sway referenced surface and visual surround.( Figure 1) The SOT 13 tests were given twice and the more difficult SOT 4-6 tests were given three times. The average of the two trials in SOT 1-3 and of the three trials in SOT 4-6 was calculated. In CDP, the subject has to maintain standing for at least 20 seconds. There is also a composite SOT score which is the weighted average of the scores of all sensory conditions, calculated as follows: (mean(SOT1)+mean(SOT2) + $3 *$ mean $($ SOT3)+3*mean(SOT4)+3*mean(SOT5)+3*mean(SOT6))/14. (NeuroCom International, Clackamas, OR) 
Each of the test items are scored according to the sway, where 100 is no sway and 0 means that the subject falls. When the subject is unable to stand for 20 seconds it is noted as Loss of Balance (LOB).The patients underwent the CDP before and three months post-operatively, and the HI twice with an interval of three months. For the HI the mean value of the two trials was calculated.

\section{Statistics}

An a priori power-analysis calculated for this study indicated that at least 16 individuals in each group were needed in order to detect a 10 point difference in SOT score at $80 \%$ power and $\alpha=0.05$. Minitab 16 Statistical Software (Minitab Inc) was used for statistical calculations. Non-parametric statistics, Mann Whitney U, and Chi squared tests for unpaired data, and the Wilcoxon sign rank test and McNemar for paired data were used. Spearman's rho correlation coefficient was used for calculating correlations.

\section{Results}

The 35 patients had lower SOT scores in all six conditions ( $\mathrm{p}=0.01$ for SOT 1 for SOT 2-6 $\mathrm{p}<0.001$ ) before surgery compared to the HI. The greatest difference was in conditions 5 and 6 (Figure 2). LOB was very frequent in SOT conditions 5 and 6 in iNPH pre-operatively and thus very significantly different from the HI (Figure 3). Pre-operatively, the patients showed significantly inferior gait capacity, had lower score in the Romberg's test, and performed a lower MMSE than the HI (Table 2).Cardiovascular risk factors were more common in the patients than the HI, though they did not reach statistical significance.

Of the 34 patients who underwent shunt surgery 20 patients could be evaluated both pre-operatively and three months post-operatively. Reasons for not being evaluated post-operatively were: five hematomas (subdural, epidural/subdural and intracerebral) post-operatively, three shunt dysfunctions (two proximal and one distal), three patients refused either surgery (one) or a second posturography 
(two), three were not evaluated a second time due to logistics reasons, and finally, one had a negative extended lumbar drainage and was not operated on.

There was a significant improved composite score $(\mathrm{p}<0.05)$ but no change in any of the SOT conditions (Figure 4) after shunt surgery. LOB was not reduced significantly after the shunt operation (Figure 5).

Post-operatively 18/20 (90\%) of the patients were considered shunt responders with a mean MOS increase of 26\% (5-67). They performed better in the Romberg's test but they did not change cognitively (MMSE) (Table 3).

The correlations for each SOT of the two examinations in the HI were: SOT 1: rho=0.41; SOT 2: rho=0.64; SOT 3: rho=0.49; SOT 4: rho=0.58; SOT 5: rho=0.65; SOT 6: rho=0.53

\section{Discussion}

The main findings of this study are that the iNPH-patients had significantly poorer balance than $\mathrm{HI}$ in all SOT conditions, and this was most pronounced in SOT 5 and 6, indicating a central vestibular dysfunction in iNPH patients. Loss of balance was also seen most frequently in conditions 5 and 6 . However, improvement after shunt could only be seen as a significant change of the composite SOT but not in any individual condition.

The patients who participated in this study were all diagnosed as probable iNPH according to the iNPH guidelines with slight modifications (1). There were no significant differences regarding sex and age between the patients and the HI. The patients had a larger percentage of cardiovascular risk factors, but this was not statistically significant (Table I). Balance in healthy elderly has been found to decrease with age. In a study comparing old old $(>80 y)$ and younger old $(<80 y)$ with the same methodology as used in our study, the mean values for each SOT condition were very close to the 
results from the $\mathrm{HI}$ in the present study(17). The interpretation would therefore be that our $\mathrm{HI}$ are representative of younger elderly people regarding balance.

CDP was developed by Nashner (18) and has been a standard for evaluating balance control (19). It is a method that allows the estimation of the relative contribution of somatosensory, vestibular and visual inputs to the balance. Test-retest for SOT has been found to be fair to good in older adults (20), but the best test-retest data comes from astronauts, where a statistically significant learning effect has been seen between the first and second baseline tests but not after the second test (21). The same methodology has been used for evaluating balance and risk of falls in Alzheimer's disease (22), for comparing balance between patients with Alzheimer's disease and Parkinson's disease (23), in Parkinson patients on whom Deep Brain Stimulation has been conducted (24) and also in patients with anxiety disorders (25). In our study there was generally a good agreement between the first and the second examination in the HI (Figure 2), which favours the methodology.

Gait in iNPH is characterized by small, broad-based steps with decreased step height $(6,26)$. Part of the gait impairment is related to the postural dysfunction, where a greater sway and a higher backward directed velocity have been shown on a force platform (8). In a more recent study using a force plate it could also be shown that patients suffering from normal pressure hydrocephalus had a greater sway compared to control subjects (27). Blomsterwall et al., and also Czerwosz et al. have reported that iNPH patients have less use of vision for maintaining balance. We could not confirm this result since the patients in our study did perform relatively better in the balance tests compared to the HI when sight was allowed. Our patients managed the easiest conditions; SOT 1-2 only slightly poorer than the HI, indicating a relatively preserved postural function, but from SOT 3-6 there was an increasing difference between the groups. In SOT 5-6 only a minority of the patients were able to remain standing and thus experienced LOB (Figure 2). SOT 5-6 are believed to be the best test items to detect a vestibular dysfunction, either central or peripheral (19). In other words; when the visual and proprioceptive stimuli are either absent or perturbed the patients must rely on the vestibular system in 
order to maintain balance. This could be interpreted to mean that the postural dysfunction in iNPH patients is due to impaired vestibular functions.

After shunt surgery, in eighteen patients out of twenty (90\%) there was a significant improvement in motor function, namely more than 5\% improvement in motor score (MOS). Disappointingly, no improvement in individual SOT could be seen. The only test indicating improvement was the composite SOT; however this test is less valuable since mean values are included. Thus the patients were found to be substantially better on simple motor function tests but there was only a minor improvement in SOT as well as in Romberg's test. This is contrary to the results of Blomsterwall et al. who concluded that the postural function was the symptom that improved most after shunt surgery (8); however this conclusion was based mainly on clinical bedside postural tests and not on the force plate examination, which did not show the same magnitude of improvement.

Explanations for unchanged SOT values might include the weakness of the method; that there is no improvement in postural function post-operatively; relatively small number of patients completed preand post-operative examinations. There could also be a selection bias that patients with the worst balance were those who might have had the greatest balance improvement after shunt operation but were excluded due to not being able to handle the test.

One limitation of this study is that medication was not controlled for. Another limitation is that the sway in a lateral direction was not examined because this is not included in the SOT protocol. The combination of high age and cardiovascular risk factor makes the possibility for diminished visual capacity, decreased proprioception, and worse vestibular function more likely and therefore we did not choose to specifically control for these factors as this could have led to difficulty interpreting the results.

The matter of balance disturbance in elderly hydrocephalic patients and its consequences is of interest for all who manage this kind of patient. LOB has been shown to predict falls within the next 16 months in healthy elderly volunteers (28). As we have shown, LOB is extremely common in the more 11 
difficult tests 5 and 6 in iNPH patients and might therefore be considered for use as a prognostic test for the risk of falls in order to be able to institute preventive measures.

Further larger studies are warranted to explore postural function in iNPH and to try to answer the question of whether or not there is an improvement in balance after shunt surgery.

\section{Conclusion}

For the first time CDP with the SOT protocol has been used to evaluate patients with iNPH. The patients showed a profound disturbance of postural function compared to HI, and this disturbance was probably caused by deficient central integration of vestibular function. There was a weak improvement in balance three months post-operatively.

\section{Acknowledgements:}

Physiotherapist Johanna Rydja and Occupational therapist Katarina Owen are kindly acknowledged for their assessments of motor and cognitive function, respectively.

Vestibular assistant Lisbeth Noaksson and Sofie Danielsson are gratefully acknowledged for examining the subjects with CDP.

Natus Medical Inc is acknowledged for the illustration in figure 1 of Sensory Organizing Test.

\section{References:}

1. Relkin N, Marmarou A, Klinge P, Bergsneider M, Black PM. Diagnosing idiopathic normal-pressure hydrocephalus. Neurosurgery. 2005;57(3 Suppl):S4-16; discussion ii-v.

2. Krauss JK, Faist M, Schubert M, Borremans JJ, Lucking $\mathrm{CH}$, Berger W. Evaluation of gait in normal pressure hydrocephalus before and after shunting. Adv Neurol. 2001;87:301-10.

3. Klinge P, Hellstrom P, Tans J, Wikkelso C. One-year outcome in the European multicentre study on iNPH. Acta Neurol Scand. 2012;126(3):145-53.

4. Brean A, Eide PK. Prevalence of probable idiopathic normal pressure hydrocephalus in a Norwegian population. Acta Neurol Scand. 2008;118(1):48-53.

5. Tanaka N, Yamaguchi S, Ishikawa H, Ishii H, Meguro K. Prevalence of possible idiopathic normal-pressure hydrocephalus in Japan: the Osaki-Tajiri project. Neuroepidemiology. 2009;32(3):171-5. 

hydrocephalus. Special reference to stance and gait. Archives of neurology. 1986;43(1):34-8.

7. Blomsterwall E, Bilting M, Stephensen H, Wikkelso C. Gait abnormality is not the only motor disturbance in normal pressure hydrocephalus. Scandinavian journal of rehabilitation medicine. 1995;27(4):205-9.

8. Blomsterwall E, Svantesson U, Carlsson U, Tullberg M, Wikkelso C. Postural disturbance in patients with normal pressure hydrocephalus. Acta Neurol Scand. 2000;102(5):284-91. 9. Sturnieks DL, St George R, Lord SR. Balance disorders in the elderly. Neurophysiol Clin. 2008;38(6):467-78.

10. Romberg MH. Lehrbuch der Nervenkrankenheiten des Menschen. 1846.

11. Tinetti ME, Williams TF, Mayewski R. Fall risk index for elderly patients based on number of chronic disabilities. The American journal of medicine. 1986;80(3):429-34.

12. Berg K, Norman KE. Functional assessment of balance and gait. Clin Geriatr Med. 1996;12(4):705-23.

13. Mancini M, Horak FB. The relevance of clinical balance assessment tools to differentiate balance deficits. European journal of physical and rehabilitation medicine. 2010;46(2):239-48.

14. Chaudhry H, Bukiet B, Ji Z, Findley T. Measurement of balance in computer posturography: Comparison of methods--A brief review. Journal of bodywork and movement therapies. 2011;15(1):82-91.

15. Mathias S, Nayak US, Isaacs B. Balance in elderly patients: the "get-up and go" test. Arch Phys Med Rehabil. 1986;67(6):387-9.

16. Folstein MF, Folstein SE, McHugh PR. "Mini-mental state". A practical method for grading the cognitive state of patients for the clinician. J Psychiatr Res. 1975;12(3):189-98.

17. Camicioli R, Panzer VP, Kaye J. Balance in the healthy elderly: posturography and clinical assessment. Archives of neurology. 1997;54(8):976-81.

18. Nashner LM, Peters JF. Dynamic posturography in the diagnosis and management of dizziness and balance disorders. Neurologic clinics. 1990;8(2):331-49.

19. Black FO. What can posturography tell us about vestibular function? Annals of the New York Academy of Sciences. 2001;942:446-64.

20. Ford-Smith CD, Wyman JF, Elswick RK, Jr., Fernandez T, Newton RA. Test-retest reliability of the sensory organization test in noninstitutionalized older adults. Arch Phys Med Rehabil. 1995;76(1):77-81.

21. Paloski WH, Black FO, Reschke MF, Calkins DS, Shupert C. Vestibular ataxia following shuttle flights: effects of microgravity on otolith-mediated sensorimotor control of posture. The American journal of otology. 1993;14(1):9-17.

22. Suttanon P, Hill KD, Said CM, Logiudice D, Lautenschlager NT, Dodd KJ. Balance and mobility dysfunction and falls risk in older people with mild to moderate Alzheimer disease. American journal of physical medicine \& rehabilitation / Association of Academic Physiatrists. 2012;91(1):1223.

23. Chong RK, Horak FB, Frank J, Kaye J. Sensory organization for balance: specific deficits in Alzheimer's but not in Parkinson's disease. The journals of gerontology Series A, Biological sciences and medical sciences. 1999;54(3):M122-8.

24. Ondo WG, Almaguer M, Cohen H. Computerized posturography balance assessment of patients with bilateral ventralis intermedius nuclei deep brain stimulation. Movement disorders :

official journal of the Movement Disorder Society. 2006;21(12):2243-7.

25. Jacob RG, Redfern MS, Furman JM. Space and motion discomfort and abnormal balance control in patients with anxiety disorders. Journal of neurology, neurosurgery, and psychiatry. 2009;80(1):74-8. 
26

Stolze H, Kuhtz-Buschbeck JP, Drucke H, Johnk K, Diercks C, Palmie S, et al. Gait analysis in idiopathic normal pressure hydrocephalus--which parameters respond to the CSF tap test? Clinical neurophysiology : official journal of the International Federation of Clinical Neurophysiology. 2000;111(9):1678-86.

27. Czerwosz L, Szczepek E, Blaszczyk JW, Sokolowska B, Dmitruk K, Dudzinski K, et al. Analysis of postural sway in patients with normal pressure hydrocephalus: effects of shunt implantation. European journal of medical research. 2009;14 Suppl 4:53-8.

28. Buatois S, Gueguen R, Gauchard GC, Benetos A, Perrin PP. Posturography and risk of recurrent falls in healthy non-institutionalized persons aged over 65. Gerontology. 2006;52(6):34552. 
Figure 1. Illustration of the Sensory Organizing Test (SOT 1-6)

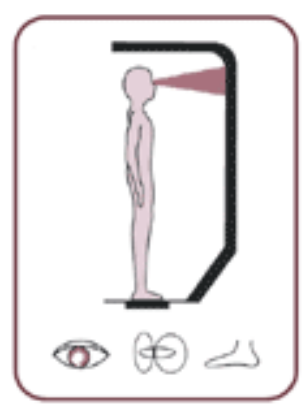

2.

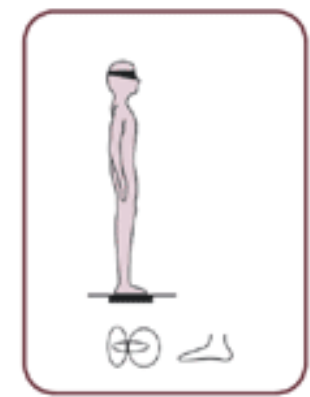

5.

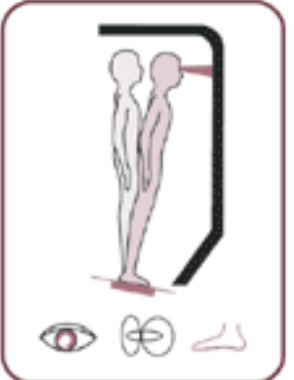

3.

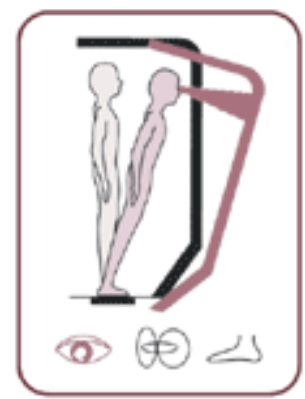

6.

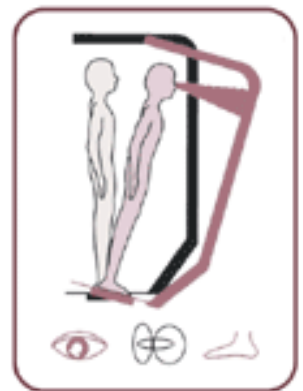

Image courtesy of Natus Medical Inc. 
Figure 2. Sensory Organizing Test (SOT). Comparison is made between the mean SOT score for HI-1 and HI-2 (the first and second examinations) and the pre-operative SOT score for iNPH. Values are given as median. Interquartile range is indicated. $* *=\mathrm{p}<0.01, * * * \mathrm{p}<0,001$.

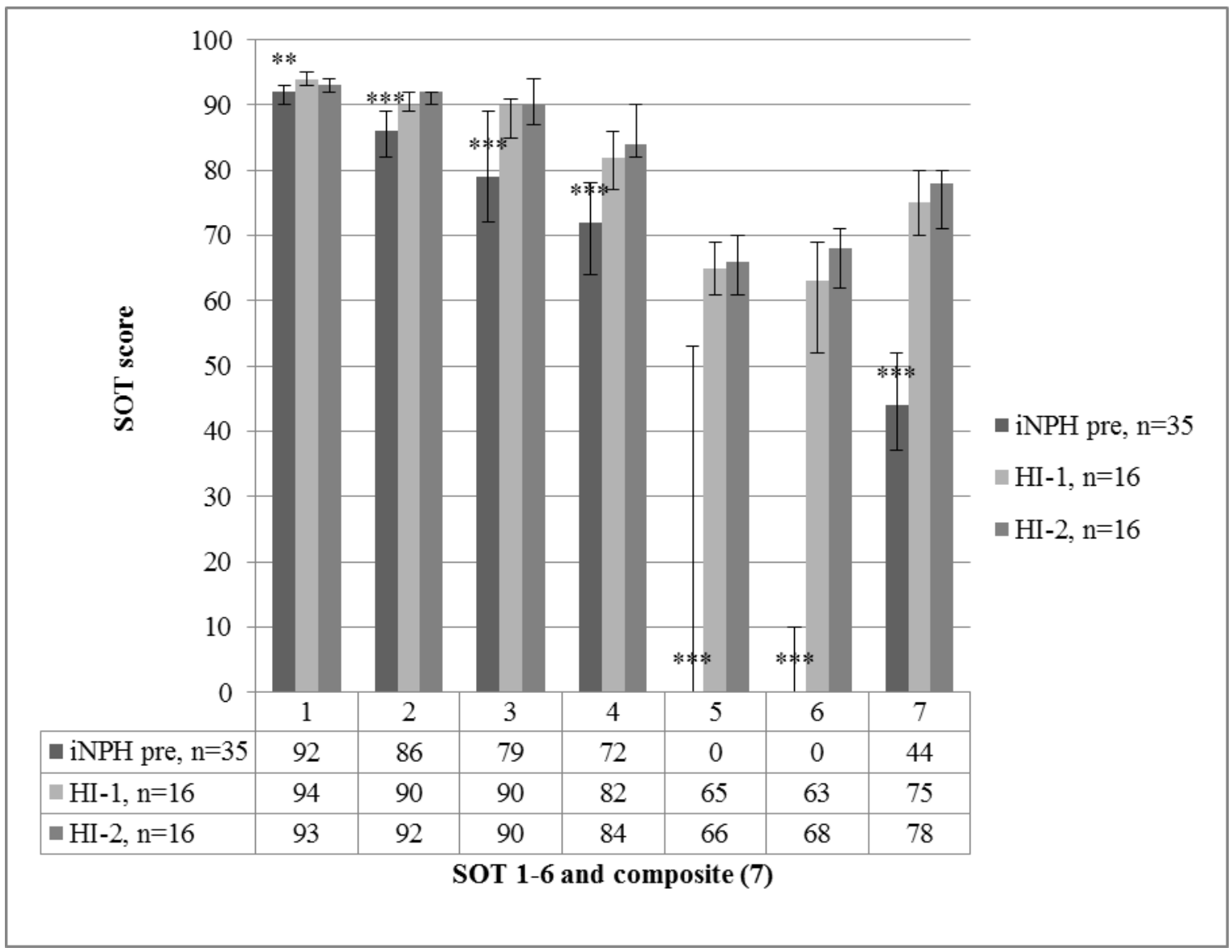


Figure 3. Loss of Balance (LOB) for each SOT condition for iNPH pre-operatively versus HI-1 and HI-2 (the first and second examinations) respectively. $* * *=p<0.001$




Figure 4. Sensory Organizing Test for iNPH pre vs. post-operatively. Median and interquartile ranges are given. $*=p<0.05$.

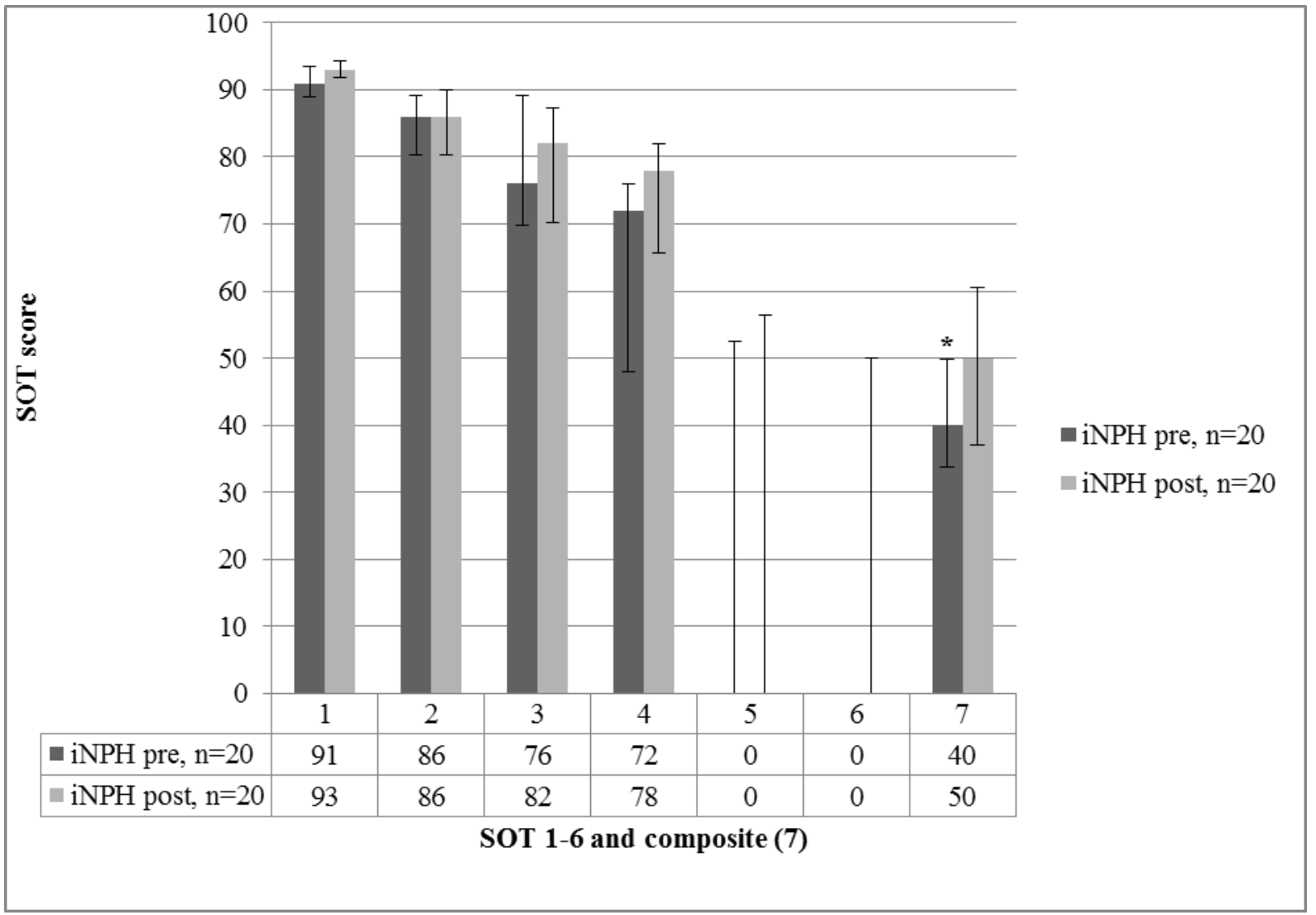


Figure 5. Loss of Balance (LOB) for each SOT condition for iNPH pre-operatively vs. iNPH post-operatively. No significant change of LOB pre- and post-operatively.

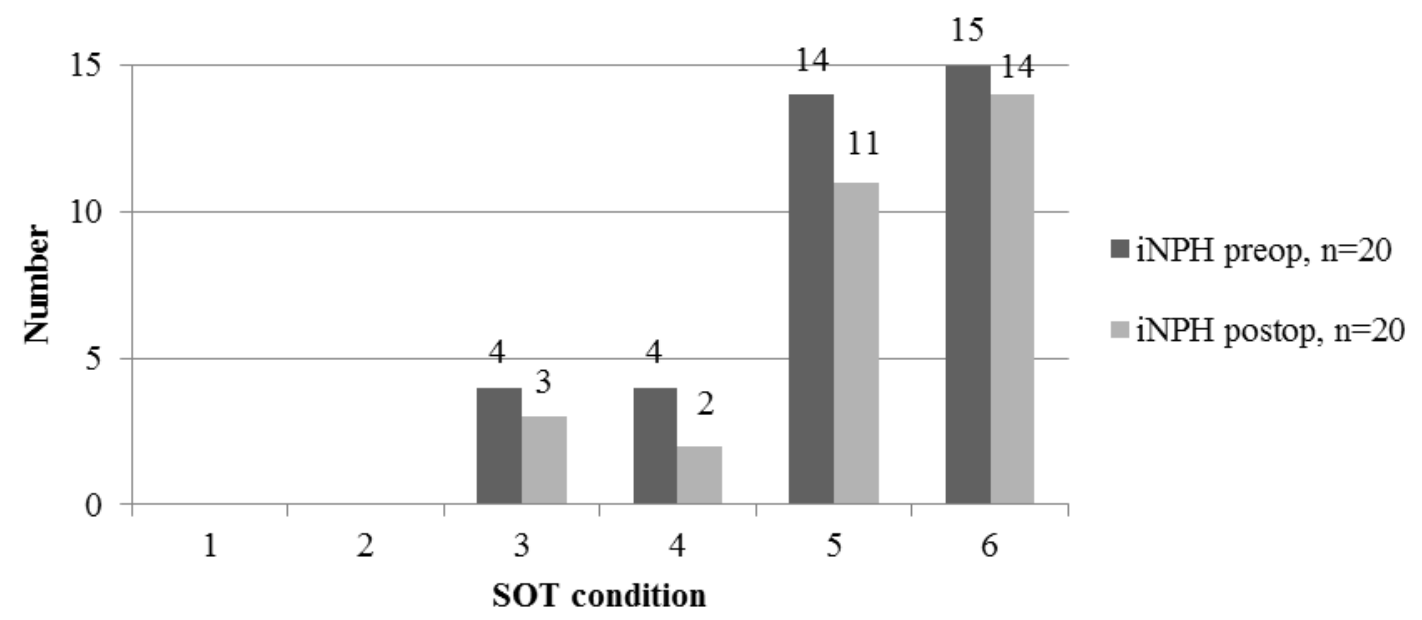


Table 1. Clinical data for iNPH vs. HI.

\begin{tabular}{|c|c|c|}
\hline & iNPH & HI \\
\hline & $n=35$ & $n=16$ \\
\hline Male/female & $16 / 19$ & $7 / 9$ \\
\hline Age (mean years, range) & $73(49-81)$ & $73(62-89)$ \\
\hline Disease duration (mean months, range) & $36(6-120)$ & N/A \\
\hline \multicolumn{3}{|l|}{ Cardiovascular risk factors } \\
\hline Hypertension & 17 & 4 \\
\hline Diabetes Mellitus & 10 & 3 \\
\hline Stroke & 7 & 1 \\
\hline Heart disease & 9 & 1 \\
\hline
\end{tabular}


Table 2. Clinical data for iNPH pre-operatively vs. HI. Values are given as median and range. $* * *=\mathrm{p}<0.001$

\begin{tabular}{|c|c|c|}
\hline & $\begin{array}{l}\text { iNPH pre } \\
\qquad \mathbf{n = 3 5}\end{array}$ & $\begin{array}{c}\text { HI } \\
\mathrm{n}=16\end{array}$ \\
\hline \multicolumn{3}{|l|}{ Gait } \\
\hline $10 \mathrm{~m}$ ( seconds) & $13(7.5-50)$ & $7(5-10) * * *$ \\
\hline $10 \mathrm{~m}$ (steps) & $22(11-65)$ & $10(8-14)^{* * *}$ \\
\hline $\begin{array}{l}\text { TUGt (seconds) } \\
n=34\end{array}$ & $14(8.5-55)$ & nd \\
\hline $\begin{array}{l}\text { TUGs (steps) } \\
n=34\end{array}$ & $22.5(10-43)$ & nd \\
\hline Cognition & & \\
\hline $\begin{array}{l}\text { MMSE } 0-30 \\
n=33\end{array}$ & $26(12-30)$ & $29(27-30)^{* * * *}$ \\
\hline Balance & & \\
\hline $\begin{array}{l}\text { Romberg's test (seconds) } \\
n=33\end{array}$ & $17(0-60)$ & $60(60) * * *$ \\
\hline
\end{tabular}


Table 3. Clinical data for iNPH-patients pre- and post-operatively. Values are given as median and range. $*=p<0.05, * *=p<0.01, * * *=p<0,001$

\begin{tabular}{|c|c|c|}
\hline & $\begin{array}{l}\text { iNPH pre } \\
\qquad \mathbf{n}=20\end{array}$ & $\begin{array}{l}\text { iNPH post } \\
\qquad \begin{array}{l}\mathbf{n}=\mathbf{2 0}\end{array}\end{array}$ \\
\hline \multicolumn{3}{|l|}{ Gait } \\
\hline 10 m ( seconds) & $16.5(7.5-50)$ & $11.5(6-26)^{* * *}$ \\
\hline 10 m (steps) & $26(16-65)$ & $20(14-31)^{* * *}$ \\
\hline TUGt (seconds) & $20(8.5-55)$ & $13.5(7-34)^{* * *}$ \\
\hline TUGs (steps) & $26.5(13-43)$ & $19(12-37) * *$ \\
\hline \multicolumn{3}{|l|}{ Cognition } \\
\hline $\begin{array}{l}\text { MMSE } 0-30 \\
n=19\end{array}$ & $27(12-30)$ & $27(16-30)$ \\
\hline \multicolumn{3}{|l|}{ Balance } \\
\hline Romberg's test (seconds) & $30(0-60)$ & $37(0-60)^{*}$ \\
\hline
\end{tabular}

